\title{
MOVIMENTI PERIODICI DI UN SOLIDO PESANTE PROSSIMI A PRECESSIONI REGOLARI.
}

\author{
$d i$ IDDA AVENANTI.
}

\section{1. - Richiamo del caso di Lagrange.}

Si abbia un corpo solido, pesante, che ruota intorno ad un punto fisso $O$. Per lo studio del movimento, riferiamoci a tre assi fissi $O\left(x^{\prime}, y^{\prime}, z^{\prime}\right)$, di cui l' asse $O z^{\prime}$ lo supporremo verticale. Riferiamoci inoltre a tre assi mobili $O(\xi, \eta$, $\zeta)$, rigidamente collegati al corpo, supponendo in particolare che questi tre assi siano gli assi principali di inerzia relativi al punto 0 .

Mettiamoci ancor più specificamente nel caso in cui l'elissoide d' inerzia, relativo al punto fisso, è di rivoluzione, nel qual caso (con le solite notazioni) $\mathbf{A}=\mathrm{B}$; e consideriamo il caso che il centro di gravità $G\left(\xi_{0}, \eta_{0}, \zeta_{0}\right)$ del corpo, si trovi nell' asse di rivoluzione $\left(\xi_{0}=\eta_{0}=0\right)$. Questo caso ̀े stato trattato da Lagrange, ed è stato completamente risoluto. Nel movimento del corpo, l'asse $O \xi$ si sposta restando sempre compreso però entro lo spazio limitato da due falde coniche di rotazione attorno alla verticale $O z^{\prime}$, col vertice comune in $O$, e le cui ampiezze $\vartheta_{1}, \vartheta_{2}$, rappresentan $\Theta$ i valori estremi fra i quali oscilla l' angolo $\widehat{\zeta O z^{\prime}}=\mathscr{V}$ (angolo di nutazione). Allorchè per determinate condizioni iniziali risulta $\vartheta_{1}=\vartheta_{,}$, il moto del corpo (o moto del giroscopio simmetrico pesante) è detto di precessione regolare. In questo caso 1' asse di figura del corpo ruota con velocità angolare costante attorno alla verticale (asse di precessione) e descrive un cono rotondo, mentre il corpo ruota con velocita angolare costante, intorno al proprio asse.

Quando il moto del corpo ̀̀ una precessione regolare, abbiamo in corrispondenza soluzioni periodiche. 


$$
\text { 2. - Caso generale. }
$$

Criterio per Ia ricerca di soluzioni periodiche.

Prendiamo ora in considerazione il problema generale della rotazione di un corpo solido pesante, attorno ad un suo punto fisso, in cui le quantità $A-B, \xi_{0}, \eta_{0}$, non sono più nulle. Ci proponiamo di discutere le soluzioni periodiche del nostro problema, per valori sufficientemente piccoli delle quantità suaccennate.

Più precisamente ci proponiamo di applicare la classica teoria di Poincarè partendo dalle precessioni regolari, le quali si possono appunto risguardare come speciali soluzioni periodiche, corrispondenti ai ralori nulli dei parametri $A-B$, $\xi_{0}, \eta_{0}$.

Chiameremo d'ora in poi questi parametri con $\mu_{1}, \mu_{2}, \mu_{3}$; e quando diremo $\mu=0$ o $\mu \neq 0$, intendercmo uguali a zero tanto $\mu_{1}$ che $\mu_{3}$ che $\mu_{3}$, o diverso da zero almeno uno dei tre.

\section{3. - Angoli di Eulero.}

Formule varie - Equazioni del moto sotto forma enuonica.

Avviamoci a mettere le equazioni del moto sotto forma canonica.

All' uopo introduciamo come coordinate lagrangiane, $i$ tre angoli di Eulero $\$, \uparrow, \psi$, the ci determinano la posizione del corpo rispetto agli assi fissi. Indichiamo con $p, q, r$, le proiezioni, sugli assi mobili, della velocità di rotazione $\omega$ del corpo intorno ad $O$, e con $\gamma, \gamma^{\prime}, \gamma^{\prime \prime}$, i coseni degli angoli che gli assi $O \xi, O \dot{\eta}, O \zeta$, formano con $O z^{\prime}$. Questi coseni avranno le espressioni

$$
\left\{\begin{array}{l}
\gamma=\operatorname{sen} \vartheta \operatorname{sen} \varphi, \\
\gamma^{\prime}=\operatorname{sen} \vartheta \cos \varphi, \\
\gamma^{\prime \prime}=\cos \vartheta .
\end{array}\right.
$$

Sappiamo che la forza viva del sistema ha l'espressione

$$
\mathrm{T}=\frac{1}{2}\left(\mathrm{~A} p^{2}+\mathrm{B} q^{2}+\mathrm{C} r^{3}\right),
$$


e il potenziale

$$
\mathrm{U}=\mathbf{M} g z^{\prime}
$$

(M, massa totale del corpo); e siccome $z^{\prime}=\xi_{\mathrm{o}} \gamma+\eta_{0} \gamma^{\prime}+\zeta_{0} \gamma^{\prime \prime}$, sostituendo anche a $\gamma, \gamma^{\prime}, \gamma^{\prime \prime}$, i valori (1), abbiamo

$$
\mathrm{U}=\mathrm{M} g z^{\prime}=\mathrm{M} g\left(\xi_{\mathrm{o}} \gamma^{\prime}+\eta_{0} \gamma^{\prime}+\zeta_{0} \gamma^{\prime \prime}\right)=\mathrm{U}(\vartheta, \varphi),
$$

da cui la funzione lagrangiana $\mathrm{L}=\mathrm{T}+\mathrm{C}$.

D' altra parte le relazioni che legano $p, q, r$, a $\vartheta, \varphi, \psi$, e loro derivate prime sono le seguenti:

$$
\left\{\begin{array}{l}
p=\dot{\psi} \operatorname{sen} \vartheta \operatorname{sen} \varphi+\dot{\vartheta} \cos \varphi \\
q=\dot{\psi} \operatorname{sen} \vartheta \cos \varphi-\dot{\vartheta} \operatorname{sen} \varphi \\
r=\dot{\vartheta} \cos \vartheta+\dot{\varphi}
\end{array}\right.
$$

Accanto alle funzioni incognite $\vartheta, \uparrow, \psi$, introduciamo le variabili coniugate $p_{\ddagger}, p_{\varphi}, p_{\downarrow}$; ossia i monenti che sono così definiti

$$
\left\{\begin{array}{l}
p_{\Im}=\frac{\partial \mathrm{T}}{\partial \dot{\vartheta}} \\
p_{\vartheta}=\frac{\partial \mathrm{T}}{\partial \dot{\varphi}} \\
p_{\psi}=\frac{\partial \mathrm{T}}{\partial \dot{\psi}}
\end{array}\right.
$$

cia cui, in base alla (2) e sostituendo a $p, q, r$, i valori (4), (5) $\left\{\begin{array}{l}p_{\Im}=\mathrm{A} p \frac{\partial p}{\partial \dot{\xi}}+\mathrm{B} q \frac{\partial q}{\partial \dot{\jmath}}+\mathrm{C} r \frac{\partial r}{\partial \dot{\varphi}}=\mathrm{A} p \cos \varphi-\mathrm{B} q \operatorname{sen} \varphi \\ p_{\phi}=\mathrm{A} p \frac{\partial p}{\partial \dot{\varphi}}+\mathrm{B} q \frac{\partial q}{\partial \dot{\varphi}}+\mathrm{C} r \frac{\partial r}{\partial \dot{\varphi}}=\mathrm{C} r \\ p_{\downarrow}=\mathrm{A} p \frac{\partial p}{\partial \dot{\psi}}+\mathrm{B} q \frac{\partial q}{\partial \dot{\psi}}+\mathrm{C} r \frac{\partial r}{\partial \dot{\psi}}=\mathrm{A} p \gamma+\mathrm{B} q \gamma^{\prime}+\mathrm{C} r \gamma^{\prime \prime}\end{array}\right.$

Procuriamoci le A $p, \mathrm{~B} q$, C $r$, espresse in funzione delle coordinate e delle loro coniugate. Ponendo per brevità di 
scritura

$$
\sigma=\frac{p_{\psi}-p_{\varphi}}{\operatorname{sen} \vartheta} \frac{\cos \vartheta}{\vartheta}
$$

otteniamo

$$
\left\{\begin{array}{l}
\mathrm{C} r=p_{\varphi}, \\
\mathrm{A} p=p_{\Im} \cos \varphi+\sigma \operatorname{sen} \varphi ; \\
\mathrm{B} q=-p_{\Im} \operatorname{sen} \varphi+\sigma \cos \varphi
\end{array}\right.
$$

Introduciamo anche la funzione hamiltoniana $H$, che si riduce notoriamente a $\mathrm{T}-\mathrm{U}$ quando, come nel caso presente, i vincoli sono indipendenti dal tempo. Dalle (2) e (3) ho

$$
\mathrm{H}=\frac{1}{2}\left\{\frac{\mathrm{A}^{2} p^{2}}{\mathrm{~A}}+\frac{\mathrm{B}^{2} q^{2}}{\mathrm{~B}}+\frac{p_{\phi}^{2}}{\mathrm{C}}\right\}-\mathrm{U}(\vartheta, \varphi),
$$

e siccome nelle espressioni (5) di A $p, \mathrm{~B} q, \mathrm{C}^{\prime} r$, non comparisce esplicitamente la variabile $\psi$, la (7) ci definisce la $\mathrm{H}$ come funzione dei soli cinque argomenti $\vartheta, \varphi, p_{\Im}, p_{\varphi}, p_{\downarrow}$. Il sistema canonico od hamiltoniano da considerare è il seguente :

$$
\left\{\begin{array}{l}
\dot{\vartheta}=\frac{\partial \mathrm{H}}{\partial p_{\Im}} \quad, \quad \dot{p}_{\Im}=-\frac{\partial \mathrm{H}}{\partial \vartheta}, \\
\dot{\varphi}=\frac{\partial \mathrm{H}}{\partial p_{\varphi}} \quad, \quad \dot{p}_{\varphi}=-\frac{\partial \mathrm{H}}{\partial \varphi} \\
\dot{\psi}=\frac{\partial \mathrm{H}}{\partial p_{\psi}}, \dot{p}_{\psi}=-\frac{\partial \mathrm{H}}{\partial \psi} . \\
4 .- \text { Integrali primi. }
\end{array}\right.
$$

\section{Riduzione consentita dall' Integrale delle aree.}

I teoremi generali sul moto dei sistemi, ci assicurano che il sistema (8) deve ammettere due integrali primi : l'integrale delle aroe e quello delle forze vive che è notoriamente $\mathrm{T}-\mathrm{U}=h(h$ costante delle forze vive $)$ ossia $\mathrm{H}=h$. Occupiamoci del primo. Abbiamo detto che la $H$ non con. tiene $\psi$, quindi nella sesta equazione, $\dot{p}_{\psi}=-\frac{\partial \mathrm{H}}{\partial \psi}$, il secondo 
membro è zero, da cui $p_{\psi}=$ costante $=\mathrm{K}$. Possiamo riconoscere che $\mathrm{K}$ è la costante delle aree. Infatti si sa che per un solido pesante il momento delle quantita di moto, rispetto alla verticale $\mathrm{O} z^{\prime}$, ciò̀ il trinomio $\mathrm{A} p \gamma+\mathrm{B} q \gamma^{1}+\mathrm{C} r \gamma^{\prime \prime}$, è costante (costante delle aree)

$\mathrm{A} p \gamma+\mathrm{B} q \gamma^{\prime}+\mathrm{C} r \gamma^{\prime \prime}=\mathrm{K}$;

ma per I' ultima delle (5) tale trimomio non è che $p_{\psi}$, quindi il suo valore costante $K$, si interpreta come costante delle aree. Vediamo come questo integrale primo consenta di abbassare l' ordine del sistema (8) di due unità, a meno di una quadratura. Immaginiamo all' uopo di sostituire a $p_{\psi}$ il suo valore costante $K$ nelle prime quattro equazioni (8). Queste ci si presentano allora come un sistema canonico ridotto relativo alle due coppie coniugate $\left(\begin{array}{cc}\vartheta & \varphi \\ p_{\Im}, & p_{\downarrow}\end{array}\right)$ :

$$
\left\{\begin{array}{c}
\dot{\vartheta}=-\frac{\partial \mathrm{H}}{\partial p_{\Im}}, \quad \dot{\varphi}=\frac{\partial \mathrm{H}}{\partial p_{\vartheta}}, . \\
\dot{p}_{\sqsupset}=-\frac{\partial \mathrm{H}}{\partial \vartheta}, \quad \dot{p}_{p}=-\frac{\partial \mathrm{H}}{\partial \varphi} .
\end{array}\right.
$$

Qualora fosse integrato questo sistema ridotto, si potrebbe riprendere la quinta delle (8) $\frac{\partial \mathrm{H}}{\partial p_{\psi}}=\dot{\psi}$, e sostituendovi nel secondo membro al posto di $\vartheta, \varphi, p_{\vartheta}, p_{\uparrow}$ i loro valori in funzione di $t$ ottenuti dall' integrazione del sistema ridotto (10), si otterrebbe la $\psi$ con una quadratura, la $p_{\psi}$ avendo d'altra darte il valore costante $K$.

Basta quindi che noi ci occupiamo del sistema ridotto (10), al quale si trasporta automaticamente l' integrale delle forze vive ponendovi $p_{\downarrow}=\mathrm{K}$. Noi potremo perciò abbassare ulteriormente il nostro sistema ridotto di due unità, mediante l' integrale $\mathrm{H}=h$. Per eseguire effettivamente l' abbassamento, conviene eliminare il tempo, assumendo una delle coordinate, e precisamente la $\varphi$, come variabile indipendente. Ciò è legittimo ogni qualvolta $\dot{\varphi}$ si mantiene di- 
versa da zero per tutti i valori del tempo (in corrispondenza alla soluzione, o alle soluzioni che si devono prendere in considerazione).

\section{5. - Ritorno alle precessioni regolari. \\ Defnizione cinematien. - Costanan della $\dot{\varphi}$. Valori di $\vartheta$ e $p_{y}$ in funzione di $\varphi$.}

Nel moto di precessione regolare, sappiamo che l' angolo $\vartheta$ resta costante e $\dot{\mathfrak{q}}$ perciò è nulla (è nulla ciò̀ la nutazione dell'asse del corpo). Il vettore $\omega$ (la velocità di rotazione) è in tal caso risultante di due vettori: l' uno diretto secondo la verticale $O z^{\prime}$ misurato da $\dot{\psi}$ in Junghezza e senso, che rappresenta la velocità di precessione dell' asse del giroscopio; l'altro diretto secondo $O_{\zeta}$, misurato da $\dot{\varphi}$ in lunghezza e senso, che rappresenta la velocità di rotazione del giroscopio intorno al proprio asse. In una precessione regolare tanto $\dot{\psi}$ che $\dot{\varphi}$ sono costanti. Si ha pertanto come condizione carateristica della precessione regolare

$$
\omega=\dot{k}+\dot{k} \chi
$$

essendo $\mathbf{k}$ e $\chi$ rispettivamente $i$ vettori unitari sulla verticale $O z^{\prime}$ e sull' asse del giroscopio $O \zeta$. Proiettando $\omega$ sullasse $O \zeta$ si ha in conformità

$$
r=\dot{\psi} \gamma^{\prime \prime}+\dot{\varphi}
$$

ma $\dot{\psi}$ e $\dot{\varphi}$ sono costanti, $\gamma^{\prime \prime}=\cos \vartheta=$ costante; quindi anche $r=$ costante $=r_{0}$.

Dacchè in una precessione regolare la $\dot{\varphi}$ è una costante, basterà supporla, come faremo, diversa da zero (il che dipende esclusivamente dalle condizioni iniziali), per poter risguardare $\varphi$, in luogo di $t$, come variabile indipendente nello studio di un tale movimento. Ora i movimenti di cui vogliamo occuparci, pur non essendo precessioni regolari, sono periodici e prossimi alle precessioni regolari; se i parametri variano di abbastanza poco, la $\dot{\varphi}$ si conserverà nell' inter- 
vallo di un periodo, e quindi indefinitamente, diversa da zero. Ciò posto, diviene lecito anche per la nosfra ricerca di immaginare senz'altro sostituita la $\varphi$ alla $t$, come variabile indipendente. Si raggiunge così il vantaggio di eliminare $d t$ e si rieavano le due equazioni relative a $\vartheta$ e a $p_{\vartheta}$ dividendo le analoghe del sistema (10) per $\frac{d \varphi}{d t}=\frac{\partial H}{\partial p_{\varphi}}$. Otterremo :

(14)

$$
\left\{\begin{array}{l}
\frac{d \vartheta}{d \varphi}=\frac{\begin{array}{l}
\partial \mathrm{H} \\
\frac{\partial p_{\vartheta}}{\partial \mathrm{H}}
\end{array}}{\frac{\partial p_{\varphi}}{\partial \mathrm{H}}} \\
\frac{d p_{\Im}}{d \varphi}=-\frac{\frac{\partial \vartheta}{\partial p_{\varphi}}}{\frac{\partial \mathrm{H}}{\partial p_{\varphi}}}
\end{array} .\right.
$$

Queste equazioni sono naturalmente applicabili anche alle precessioni regolari. Essendo in esse $\vartheta=$ cost e $\psi$ e $\varphi$ funzioni lineari del tempo, si riconosce subito che $p_{9}=0$. Infatti per la prima delle (5) si ha nel caso attuale (in cui $A=B$ )

$$
p_{\sqsupset}=\mathrm{A}(p \cos \varphi-q \operatorname{sen} \varphi)
$$

dalla quale, sostituendo a $p$ e $q$ i loro valori (4) in eui sia posto $\dot{\mathfrak{j}}=0$, risulta appunto $p_{9}=0$. Il sistema (16) è dunque certamente soddisfatto dalla soluzione periodica $\left\{\begin{array}{l}\vartheta=\vartheta_{0} \\ p_{9}=0\end{array}\right.$, che per quanto precede corrisponde ad una precessione regolare.

\section{6. - Ulteriore riduzione ad un solo grado di liberta.}

Riprendiamo 1' integrale delle forze vive

$$
\mathbf{H}\left(p_{\ni}, p_{\varphi}, \mathrm{K}, \uparrow, \varphi\right)=h,
$$

e ricaviamo da questa equazione implicita la $p_{\bullet}$, ciò che è certo legittimo perehè, come si ̀̀ visto poco fa, per le soluzioni che ci interessano, $\frac{\partial \mathrm{H}}{\partial p_{\diamond}}=\dot{\varphi}$ ̀े diversa da zero. 
Indichiamo per maggior chiarezza con

$$
p_{\varphi}=\mathbf{F}\left(p_{\ni}, \vartheta, \varphi, \mathbf{K}\right),
$$

l'equazione risoluta. Se sostituisco questo valore nella $\mathrm{H}=h$, ho una identita ; e se indico con $\alpha$ uno qualsiasi degli argomenti da cui dipende la $p_{\varphi}$, ho che

$$
\frac{\partial \mathrm{H}}{\partial \alpha}+\frac{\partial \mathrm{H}}{\partial p_{0}} \frac{\partial \mathrm{F}}{\partial \alpha}=0
$$

per cui possiamo dire che $i$ rapporti che figurano nei secondi membri del sistema $(\mathbf{1 6})$, si presentano come $-\frac{\partial \mathrm{F}}{\partial p_{s}}$, $\frac{\partial F}{\partial q}$. Posso così scrivere il sistema (16) come segue :

$$
\left\{\begin{array}{l}
\frac{d \vartheta}{d \varphi}=-\frac{\partial \mathrm{F}}{\partial p_{\Im}} \\
\frac{\partial p_{\Im}}{\partial \varphi}=\frac{\partial \mathrm{F}}{\partial \vartheta}
\end{array}\right.
$$

Questo sistema vale (con le riferite limitazioni qualitative) per $\mu$ qualunque, quindi in particolare anche per $\mu=0$. Il generale la $F$ contiene esplicitamente la $\varphi$, ed esso sistema (I) non ammette l' integrale $\mathrm{F}=$ cost. Questa circostanza si presenta per il caso particolare $\mu=0$, risultando allora $\mathrm{F}$ indipendentemente da $\varphi$.

E per la prima delle $\left(5^{\prime}\right) \quad \mathrm{O} r=p_{\varphi}$, noi rileviamo anche 1' interpretazione meccanica del valore costante F. Abbiamo insomma per $\mu=0$ anche un terzo integrale

$$
\mathrm{F}=\mathrm{C} \boldsymbol{r}_{0} \text {. }
$$

\section{7. - Richiamo del teorema generale di esistenza.}

Tutto ciò premesso, riportiamoci alla teoria generale di Poincaré concernente le soluzioni periodiche di un sistema differenziale dipendente da uno o più parametri $\mu$. Se del sistema si conosce una soluzione $\pi_{0}$, corrispondente allo speciale valore $o$ agli speciale valori $\mu=0$ dei parametri, $\theta$ se 
inoltre i moltiplicatori $\left.\rho^{\prime}\right)$ di tale soluzione sono tutti diversi da uno, allora il sistema ammette soluzioni periodiche prossime a $\pi_{0}$ per ogni valore (abbastanza piccolo) dei parametri $\left.\mu^{2}\right)$. Applichiamo questa proposizione al sistema (I). Per $\mu=0$ esso ammette la soluzione (precessione regolare)

$$
\vartheta=\vartheta_{\bullet}, \quad p_{\Im}=0
$$

costituita, come si vede, da valori costanti dei due argomenti $\vartheta, p_{\vartheta}$, i quali si possono risguardare come funzioni periodiche di $\varphi$ di periodo qualsiasi, in particolare di periodo $2 \pi$ che è quello spettante ai secondi membri delle (I) per $\mu$ qualunque. Sarà questa la nostra $\pi_{0}$. Dobbiamo determinarne i moltiplicatori. Si potrebbe all'uopo sfruttare la circostanza favorevole che del sistema (I) si conosce, per $\mu=0$, l'integrale generale (rotazioni di un solido pesante nel caso di Lagrange), nel qual caso la discussione dell' esistenza di soluzione periodiche prossime a $\pi_{0}$, richiede soltanto operazioni in termini finiti ${ }^{3}$ ). Ma siccome l' espressione dell' integrale generale suaccennato, involge le funzioni ellittiche, si arriva più comodamente allo scopo seguendo la via generale (quella che conviene battere quando, anche per $\mu=0$, si conosce soltanto la soluzione $\pi_{0}$ ), la quale consiste nel formare le equazioni alle variazioni a partire da $\pi_{0}$, per determinarne gli esponenti caratteristici, o ciò chè è sostanzialmente la stessa cosa, i moltiplicatori. Nel caso attuale il

1) È appena necessario ricordare che, se $2 \pi$ è il periodo e $\alpha$ un espononte caratteristico, si chiama moltiplicatore il numero $\rho=e^{\frac{\alpha}{2 \pi}}$, che e appunto il coefficiente per cui viene moltiplicata in capo ad un periodo, quella delle soluzioni, o ciascuna se ve ne ha più, infinitamente vicina e $\pi_{0}$ i cui elementi sono del tipo $e^{\alpha \varphi} \times$ funzione periodica $\varphi, \varphi$ designando come nel nostro caso, la variabile indipendente.

2) Cfr. Poincarè * Méthodes nouvelles de la mécanique céleste ». [T. I, p. 181].

') Cfr. per es. Picard. « Traite d'analyse*. T. III, (2.a edizione 1901, p. 170). 
procedimento riesce particolarmente semplice, perchè da un lato i secondi membri delle ( $\mathrm{I}$ ), in corrispondenza a $\mu=0$, non dipendono esplicitamente da $\varphi$, e dall' altro nella soluzione $\pi_{0}, \vartheta$ e $p_{0}$ sono costanti. Se si pone

$$
\left\{\begin{array}{l}
\vartheta-\vartheta_{0}=\xi, \\
p_{\Im}=\eta,
\end{array}\right.
$$

e si trattano $\xi$ e $\eta$ come infinitesimi, le equazioni alle variazioni provenienti dalle (I) che definiscono $\xi$ e $\eta$, risultano evidentemente a coeffieienti costanti. Anche per questa via il ealcolo dei moltiplicatori si trova in sostanza ricondotto ad operazioni algebriche : risoluzione dell' equazione caratteristica, nel caso nostro appena di secondo grado, che ha per radici i moltiplicatori.

Passiamo alla esecuzione effettiva dei calcoli.

\section{8. - Equarieni alle variazioni. - Determinaxione del coeffelenti.}

Le equazioni alle variazioni del sistema (I) a partire dalla soluzione $\pi_{0}$, in base alla posizione (19), sono:

$$
\left\{\begin{array}{c}
\frac{d \xi}{d \varphi}=-\frac{\partial^{2} \mathrm{~F}}{\partial \vartheta^{2} \partial p_{\vartheta}} \xi-\frac{\partial^{2} \mathrm{~F}}{\partial \vartheta^{2}} \eta, \\
\frac{d \eta}{d \varphi}=\frac{\partial^{2} \mathrm{~F}}{\partial \vartheta^{2}} \xi+\frac{\partial^{2} \mathrm{~F}}{\partial p_{9} \partial \vartheta^{2}} \eta,
\end{array}\right.
$$

dove, ben s' intende, nei secondi membri si deve porre a derivazione eseguita $\checkmark=J_{0}, p_{\Im}=0$.

Per esplicitare le derivate seconde della $F$, ricordiamo che la $F$ deve intendersi definita in funzione di $s^{\text {a }} p_{5}$ dalla equazione implicita (17) $\mathrm{H}=h$, la quale, attesa la espressione che compete ad $\mathrm{H}$ per $\mu=0\left(\operatorname{ciò} A=B, \xi_{0}=\eta_{0}=0\right)$, in base alle (7), (5') e (3), assume l' aspetto

$$
\mathrm{H}=\frac{1}{2 \mathrm{~A}}\left\{p_{9}^{2}+\sigma^{2}\right\}+\frac{1}{2 \mathrm{C}} \mathbf{F}^{2}-\mathbf{M} g \xi_{0} \cos \mathrm{J}=h \text {. }
$$


Se, come già a $\S 6$, designamo per brevità con $\alpha(0 \beta)$ nno qualunque dei due argomenti $s$ o $p_{\text {s }}$, e se inoltre poniamo per brevità di scrittura

$$
\begin{gathered}
\mathrm{F}_{\alpha}=\frac{\partial \mathrm{F}}{\partial \alpha} \quad, \mathrm{F}_{\beta}=\frac{\partial \mathrm{F}}{\partial \beta}, \mathrm{H}_{\alpha}=\frac{\partial \mathrm{H}}{\partial \alpha}, \mathrm{H}_{\beta}=\frac{\partial \mathrm{H}}{\partial \beta}, \\
\mathrm{H}_{0}=\frac{\partial \mathrm{H}}{\partial \mathrm{F}}, \mathrm{H}_{00}=\frac{\partial^{2} \mathrm{H}}{\partial \mathrm{F}^{2}}, \mathrm{H}_{0 x}=\frac{\partial^{2} \mathrm{H}}{\partial \mathrm{F} \partial \alpha}, \mathrm{H}_{0 \beta}=\frac{\partial^{2} \mathrm{H}}{\partial \mathrm{F} \partial \beta}, \\
\mathrm{H}_{\alpha \beta}=\frac{\partial^{2} \mathrm{H}}{\partial \alpha \partial \beta} \quad, \quad \mathrm{F}_{\alpha \beta}=\frac{\partial^{2} \mathrm{~F}}{\partial \alpha \partial \beta}
\end{gathered}
$$

abbiamo

$$
\left\{\begin{array}{l}
\mathrm{H}_{\alpha}+\mathrm{H}_{0} \mathbf{F}_{\alpha}=0, \\
\mathrm{H}_{\beta}+\mathrm{H}_{0} \mathrm{~F}_{\beta}=0 .
\end{array}\right.
$$

Accanto a queste identita, sussistono anche quelle che se ne traggono derivando ulteriormente. Esse si compendiano in

$$
\mathrm{H}_{x \beta}+\mathrm{H}_{0 \alpha} \mathrm{F}_{\beta}+\left(\mathrm{H}_{0 \beta}+\mathrm{H}_{\mathrm{oo}} \mathrm{F}_{\beta}\right) \mathrm{F}_{\alpha}+\mathrm{H}_{0} \mathrm{~F}_{\alpha \beta}=0 \text {. }
$$

Noi abbiamo bisogno di procurarci la $F_{\alpha \beta}$ in corrispondenza ai valori $\Im=\searrow_{0}, p_{9}=0$, i quali soddisfacendo alle (I) annullano $\frac{\partial \mathrm{F}}{\partial J}, \frac{\partial \mathrm{F}}{\partial p_{\Im}}$.

Possiamo pertanto porre addirittura nella (23) $\mathrm{F}_{\alpha}=\mathrm{F}_{\beta} \Rightarrow 0$, con che risolvendo rispetto a $F_{\alpha \beta}$ si ha

$$
F_{\alpha \beta}=-\frac{H_{\alpha \beta}}{H_{0}} \text {. }
$$

Ponendovi successivamente

otterremo

$$
\alpha=\Im, \quad \beta=p_{9} ; \alpha=\beta=2 ; \alpha=\beta=p_{9},
$$

$$
\left\{\begin{array}{l}
\mathrm{F}_{9 p}=-\frac{\mathrm{H}_{9}^{p}}{\mathrm{H}_{9}}, \\
\mathrm{~F}_{9 g}=-\frac{\mathrm{H}_{99}}{\mathrm{H}_{0}}, \\
\mathrm{~F}_{p_{9} p_{9}}=-\frac{\mathrm{H}_{p_{9}} p_{9}}{\mathrm{H}_{0}} .
\end{array}\right.
$$


Siamo cosí ricondotti al calcolo materiale delle seguenti derivate di $\mathrm{H}$.

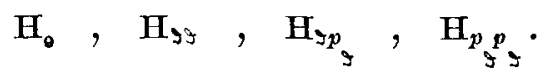

Si ha intanto

$$
\left\{\begin{array}{l}
\mathrm{H}_{0}=\frac{1}{\mathrm{~A}} \sigma \frac{\partial \sigma}{\partial \mathrm{F}}+\frac{\mathrm{F}}{c}, \\
\mathrm{H}_{\Im \Im}=\frac{1}{\mathrm{~A}}\left\{\left(\frac{\partial \sigma}{\partial^{\Im}}\right)^{2}+\sigma \frac{\partial^{2} \sigma}{\partial \Im^{2}}\right\}+M g \zeta_{0} \cos \checkmark, \\
\mathrm{H}_{p_{\Im} p_{\Im}}=\frac{1}{\mathrm{~A}}, \mathrm{H}_{\Im p_{\Im}}=0 ;
\end{array}\right.
$$

in cui a norma della (6)

$$
\sigma=\frac{\mathrm{K}-\mathrm{F} \cos \mathrm{S}}{\operatorname{sen} \mathrm{I}},
$$

e - ripetiamolo ancora una volta - a derivate eseguite si deve porre

$$
\tau=\tau_{0}, \quad p_{s}=0 \text {. }
$$

E il momento di esplicitare le derivate di $\sigma$ in base alla (26) ponendovi, a derivazione eseguita, $\Im=\Im_{0}, F=\mathrm{C} r_{0}$. Siccome poi

$$
\mathrm{H}_{\vartheta}=\frac{1}{\mathrm{~A}} \sigma \frac{\partial \sigma}{\partial \Im}+\mathrm{Mg} \zeta_{\mathrm{o}} \operatorname{sen} \Im,
$$

si annulla al pari di $F_{y}$, così sarà pure opportuno sfruttare la (27) (cioè in sostanza la ben nota relazione ") fra $i$ dati della questione e le tre costanti di integrazione $\tau_{a}, r_{0}, \mathrm{~K}$, che caratterizzano le precessioni regolari) per semplificare le espressioni finali. Si ha così :

$$
\begin{gathered}
\mathrm{H}_{0}=-\frac{1}{\mathrm{~A}} \sigma \frac{\cos \vartheta_{0}}{\operatorname{sen} \vartheta_{0}}+r_{0}, \\
\frac{\partial \sigma}{\partial \tau}=\frac{\mathrm{C} r_{0}-\mathrm{K} \cos \vartheta_{0}}{\operatorname{sen}^{2} \vartheta_{0}},
\end{gathered}
$$

1) Cfr. L. Silla. « Sopra i moti di precessione regolare del giroscopio simmetrico pesante $*$. [Rendiconti della $R$. Accademia dei Lincei, vol. XXVIII. $2 .^{\circ}$ semetre, seduta del 2 novembre 1919]. 


$$
\begin{aligned}
& \frac{\partial^{3} \sigma}{\partial \vartheta^{2}}=-\frac{2\left(\mathrm{C} r_{0}-K \cos \Im_{0}\right) \cos \Im_{0}}{\operatorname{sen}^{3} \vartheta_{0}}+\frac{K \operatorname{sen}^{2} \unlhd_{0}}{\operatorname{sen}^{3} \vartheta_{0}}= \\
& =\frac{\left(\mathrm{K}-\mathrm{C} r_{0} \cos \searrow_{0}\right)-\cos \Xi_{0}\left(\mathrm{C} r_{0}-\mathrm{K} \cos \Xi_{0}\right)}{\operatorname{sen}^{3} \vartheta_{0}}= \\
& =\frac{\sigma}{\operatorname{sen}^{2} \vartheta_{0}}-\cot \vartheta_{0} \frac{\partial \sigma}{\partial \partial} \text {. }
\end{aligned}
$$

Con ciò la espresione (25) di $H_{\Im \unlhd}$ può essere scritta come segue :

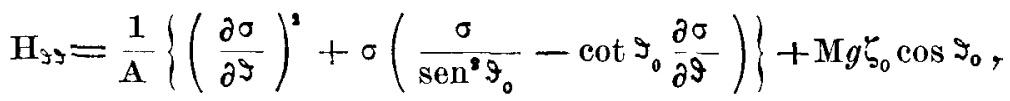

ove si sostituisca provvisoriamente ad $\mathbf{M} g \zeta_{0}$ il suo valore tratto dalla (27) e si ponga per brevità

$$
c=\frac{1}{A}\left\{\frac{\sigma^{2}}{\operatorname{sen}^{2} \vartheta_{0}}+\left(\frac{\partial \sigma}{\partial J}\right)^{2}-\frac{2 \sigma}{\operatorname{sen} J_{0}} \frac{\partial \sigma}{\partial J} \cos \Im_{0}\right\}
$$

si ottiene

$$
\left\{\begin{array}{l}
\mathrm{F}_{y \vartheta}=-\frac{c}{\mathrm{H}_{0}} \\
\mathrm{~F}_{\Im p}=0 \\
\mathrm{~F}_{p_{g} p}=-\frac{1}{\mathrm{~A} \mathrm{H}_{0}} .
\end{array},\right.
$$

La espressione (29) di c rende manifesto che questa costante è essenzialmente positiva. In base alle (30) le equazioni (20) si riducono a

$$
\left\{\begin{array}{l}
\frac{d \xi}{d \varphi}=\frac{1}{\mathbf{A H}_{0}} \eta \\
\frac{d \eta}{d \varphi}=-\frac{c}{\mathrm{H}_{0}} \xi .
\end{array}\right.
$$

La corrispondente equazione caratteristica è così

$$
\left|\begin{array}{cc}
-\rho_{j} & \frac{1}{\mathrm{AH}_{0}} \\
-\frac{c}{\mathrm{H}_{0}} & -\rho
\end{array}\right|=0
$$


ossia

$$
P^{2}+\frac{c}{A \mathbf{H}_{0}^{2}}=0
$$

Dacchè, come s'è notato or ora, $c>0$, i moltiplicatori. $\rho$ risultano immaginari puri ${ }^{1}$ ); nessuno di essi può essere pertanto uguale all' unità; e ciò basta in virtù della conclusione richiamata al $\S 7$, ad assicurare la esistenza di soluzioni periodiche prossime alla $\pi_{0}$.

Potremo dire che come nel giroscopio simmetrico esistono dei movimenti (precessioni regolari) che si possono risguardare come speciali soluzioni periodiche, anche in un giroscopio non più simmetrico, ma che differisce di poco da quello, esistono delle soluzioni periodiche alle quali corrispondono dei movimenti che potremo chiamare quasi precessioni.

\section{9. - \$tabllita - Caso di una rapida rotazione giroscopica.}

Per la stabilita (almeno in prima approssimazione) di una generica soluzione periodica è notoriamente necessario e basta, che tutti gli esponenti caratteristici siano immaginari puri, ossia che tutti $\mathrm{i}$ moltiplicatori siano in modulo uguali all' unità. Considerando in particolare le precessioni regolari $\pi_{0}$, ricaviamo dalla (31) (in base all' osservazione

1) Non sard male avvertire ohe questa circostanza ò solo apparentemente in contradizione con un teorema di Poincaré [loc. cit. pp. 179-180]. Tale teorema afferma che, se la variabile indipendente non compare esplicitamente nel sistema difforenziale (e basta che non figuri per $\mu=0$ ), allora uno almeno degli esponenti caratteristici a nullo, il che implica uno almeno dei moltiplicatori uguale all' unita. Va tenuto presente che questo risultato a essenzialmente subordinato all ipotesi che la soluzione periodioa $\pi_{0}$ da cui si parte, non sia costituita da valori tutti costanti delle inoogaite. Noi ei troviamo appunto in questo caso ecoezionale in cui il teorema non più applicabile, o infatti troviamo che non oi può essere nessun moltiplicatore uno. 
che $c$ è necessariamente $>0$ ) come condizione di stabilità, la relazione

$$
\underset{\mathrm{AH}_{0}^{2}}{c}=1
$$

Per le soluzioni periodiche prossime alla $\pi_{0}$, i moltiplicatori sono funzioni continue dei parametri $\mu$. I loro moduli possono avere pertanto il valore uno solo quando, nella precessione regolare da cui si parte, sia il rapporto $\frac{c}{\bar{A} \mathrm{H}_{0}}$ prossimo ad uno. Acquista perciò interesse l' esame delle circostanze (intrinseche ed iniziali) in corrispondenza alle quali la (32) si trova soddisfatta.

A titolo di esempio consideriamo un caso particolare notevole in cui è molto semplice la discussione ed espressiva la conclusione.

Alludiamo àl caso in cui, per la precessione regolare $\pi_{0}$ è molto rapida la rotazione attorno all' asse di simmetria : s' intende rapida rispetto all' altra rotazione attorno all' asse fisso. Ciò è quanto dire $\dot{\psi}$ va risguardata pressochè trascurabile rispetto a $\dot{p}$. Ricordiamo le espressioni (4) che ci danno $p, q, r$, in funzione di $\curvearrowright, \varphi, \psi$, e loro derivate prime:

$$
\left\{\begin{array}{l}
p=\dot{\psi} \operatorname{sen} 9 \operatorname{sen} \varphi+\dot{\jmath} \cos \varphi \\
q=\dot{\psi} \operatorname{sen} 9 \cos \varphi-\dot{\Im} \operatorname{sen} \varphi \\
r=\dot{\psi} \cos \varphi+\dot{\varphi}
\end{array}\right.
$$

Supporre la $\dot{\psi}$ trascurabile di fronte alla $\rho$ equivale (dacchè $\dot{s}=0$ ) a supporre $p, q$ trascurabili rispettto ad $r$. Se ricordiamo inoltre che $\mathrm{K}$ (costante delle aree) è espressa per la (9) da

$$
\mathrm{K}=\mathrm{A} p \gamma+\mathrm{B} q \gamma^{\prime}+\mathrm{C} r \gamma^{\prime \prime},
$$

riconosciamo che essa si riduce più semplicemente a

$$
\mathrm{K}=\mathrm{C} r_{0} \cos \unlhd_{0}
$$


ma ̀̀ $\sigma=\frac{\mathrm{K}-\mathrm{C} r_{0} \cos \Sigma_{0}}{\operatorname{sen} \mathrm{S}_{0}}$. Possiamo quindi asserire che nella rotazione rapida, $\sigma$ risulta trascurabile di fronte alla $\mathrm{F}=\mathrm{C} r_{0}$.

Con ciò le espressioni (25) di $\mathrm{H}_{0}$ e $\mathrm{H}_{\Im \vartheta}=c$ si semplificano come segue

$$
\mathrm{H}_{0}=r_{0} \quad, \quad \mathrm{H}_{9 S}=c=\frac{\mathrm{C}^{2}}{\mathrm{~A}} r_{0}^{2},
$$

e la condizione (32) di stabilità si riduce così a $\frac{C}{\mathrm{~A}}=1$.

Ne consegue che possono essere stabili, tra le nostre soluzioni periodiche del caso generale, soltanto quelle che si riattaccano per continuita alle precessioni regolari di un solido a elissoide di inerzia sferico.

\section{0. - Considerazioni riassuntive.}

\section{Inessenziale aperiodicità dell' aximut.}

Casi particolari di periodicità completa.

I risultati precedenti sono stati conseguiti ed illustrati con referenza al sistema differenziale ridotto (I), che effettivamente riassume il problema dal punto di vista analitico. Non bisogna dimenticare tuttavia che per arrivare alle (I) dalle originarie equazioni del moto, abbiamo successivamente eliminato due elementi essenziali dell' aspetto geometricocinematico della questione; ossia l'angolo di precessione $\psi$ e il tempo $t$, accontentandoci di rilevare che una volta integrato il sistema (1), la loro determinazione dipende soltanto da quadrature. Importa rendersi conto del comportamento di questi argomenti in corrispondenza alle soluzioni periodiche (di periodo $2 \pi$ rispettto $a \varphi$ ) di cui abbiamo testè dimostrata l' esistenza.

In primo luogo la relazione fra $\varphi$ e $t$ è data da

$$
\frac{d \varphi}{d t}=\frac{\partial \mathrm{H}}{\partial p_{\vartheta}} .
$$

Il secondo membro, per le nostre soluzioni periodiche, diviene una funzione periodica di $\varphi$ che non si annulla mai. 
La $t$ viene in conformità definita come funzione di $\varphi$, che si incrementa di una quantità costante

$$
\mathbf{T}=\int_{0}^{2 \pi} \frac{d \varphi}{\frac{\partial \mathrm{H}}{\partial p_{\phi}}},
$$

quando $\varphi$ si rincrementa di $2 \pi$. Ritornando a $t$ come variabile indipendente, e considerando in conformità la funzione inversa $\varphi(t)$, questa ci si presenta come periodica e dotata del periodo T. Lo stesso segue per $\checkmark$ e $p_{\Im}$ già definite come funzioni di $\varphi$ col periodo $2 \pi$. Quanto all' angolo $\psi$ che caratterizza l'orientazione del corpo attorno alla verticale, esso si deve ricavare in base alle originarie (8), mediante una ulteriore quadratura, dalla

$$
\frac{d \Psi}{d t}=\frac{\partial \mathrm{H}}{\partial p_{\psi}},
$$

dove al secondo membro va sostituita $K$ al posto di $p_{\downarrow}, \theta$ a tutte le altre lettere si debbono attribuire le espressioni corrispondenti alla soluzione peridica di cui si tratta, espressioni che si possono ormai risguardare come funzioni periodiche di $t$ col periodo $T{ }^{\prime}$ ).

La quadratura fornisce $\psi$ come funzione di $t$, la quale subisce, in capo ad un periodo $\mathrm{T}$ l' incremento costante

$$
\Psi=\int_{0}^{\mathrm{T}} \frac{d t}{\frac{\partial \mathrm{H}}{\partial p_{\dot{\zeta}}}} .
$$

In generale $\Psi$ non sarà un multiplo intero di $2 \pi$; e per conseguenza il corpo (o meglio la sua linea dei nodi) non riprenderà in capo ad un periodo, il medesimo azimut attorno

1) In verità la $\varphi$ non è periodica, ma subisce in capo ad un periodo un aumento di $2 \pi$. Però in $\frac{\partial \mathrm{H}}{\partial p_{\psi}}$ l' argomento $\varphi$ compare soltanto pel tramite delle funzioni trigonometriche seno e coseno. L' affermazione di periodicità è cosı giustificata. 
alla verticale. Le soluzioni trovate sono dunque periodiche a meno di tale orientazione assoluta nello spazio, che non ha del resto (almeno in generale) essenziale importanza quanto all' andamento generale del moto. Ci siamo messi in sostanza da questo punto di vista, ricorrendo fin da principio alla eliminazione, o come dicono gli inglesi, alla ignorazione della coordinata $\psi$. E manifesto che per la completa periodicità del moto è necessario e basta che $l^{\prime}$ angolo $\Psi$ risulti commensurabile con $2 \pi$, ossia della forma

$$
\Psi=\frac{m}{n} 2 \pi
$$

con $m, n$, interi primi fra loro. In tal caso a partire da un istante generico $t$, il corpo si ritroverebbe in capo all' intervallo di tempo $n$ T esattamente nella stessa posizione e con lo stesso atto di movimento dell' istante $t$. 